www.jmscr.igmpublication.org Impact Factor 5.244

Index Copernicus Value: 83.27 ISSN (e)-2347-176x ISSN (p) 2455-0450 crossref DOI: _https://dx.doi.org/10.18535/jmscr/v4i12.91

\title{
A Clinico - Hematological Profile of Nutritional Anaemia among Adolescent Girls in Rural Area around Karad, District- Satara, Maharashtra
}

\author{
Authors \\ Dr Pankaj Goyal, Dr V.R. Potdar, Dr B. Raghunandan Reddy \\ Krishna Institute of Medical Sciences and Deemed University Malkapur, Karad District- Satara \\ Email:drpankajgoyal2008@gmail.com
}

\begin{abstract}
Objectives: To estimate the prevalence of anemia in adolescent girls in rural area around karad and to assess the nutritional and socio demographic factors contributing to anemia among adolescent girls.

Materials and Methods: A cross sectional survey was conducted on randomly selected adolescents girls attending Zila Parishad schools in rural area of Karad district-Satara as per the inclusion and exclusion criteria. A total of 380 adolescent girls (10-19 years old) were included in this study. The study took place over 1 year from January to December 2015. Statistical analyses were done using percentage, standard error of proportion, Chi square test.

Results: The prevalence of anemia was found to be $45.3 \%$. A significant association of anemia was found with socioeconomic status and irregular menstruation. Mean height and weight of subjects with anemia was significantly less than subjects without anemia.

Conclusions: Among the factors low socio-economic status, vegetarian diet, history of deworming, presence of menstruation, irregular menstruation, heavy amount of bleeding during menstruation, more than 5 days of menstruation, menstruation associated with blood clots and underweight girls are significantly associated with the presence of anemia.
\end{abstract}

Keywords: Adolescent female, anemia, nutrition, rural, menstruation.

\section{INTRODUCTION}

Anemia (Greek "bloodlessness"), a blood condition involving an abnormal reduction in the number of red blood cells (erythrocytes) or in their haemoglobin content. Anaemia is defined as decreased concentration of haemoglobin and RBC mass as compared to the values in age-matched controls causing reduction in the oxygen carrying capacity of the blood. ${ }^{1}$.There are three primary causes: (1) reduced production of red blood cells, which may result from deficiency in nutrients or hormones, or from disease or other conditions; (2) excessive destruction of red blood cells, often a hereditary problem; and (3) excessive blood loss. Anaemia is one of the leading causes of morbidity and mortality in Pediatric age group including adolescents. As per the reports of NFHS-3 and the National Nutrition Monitoring Bureau Survey (NNMBS), over 55 per cent of adolescent girls are anaemic $^{2}$. Percentage prevalence of anaemia among adolescent girls in the age group 15-19 years remains almost stagnant at 55.8 per cent ${ }^{3}$. The term 'nutritional anemia' encompasses all pathological conditions in which the blood hemoglobin concentration drops to an abnormally 
low level, due to a deficiency in one or several nutrients. The main nutrients involved in the synthesis of hemoglobin are iron, folic acid, and vitamin B 12. In public health terms, iron deficiency is by far the most common cause of nutritional anemia worldwide. Folic acid deficiency is less widespread and is often observed with iron deficiency. Vitamin B 12 deficiency is far rare. Nutritional anemia is associated with impaired performance of a range of mental and physical functions in children and adolescents, along with increased morbidity ${ }^{4}$.

Adolescence health is of prime importance because they undergo a transition from childhood to adulthood. These teen years are a period of intense growth, not only physically, but also mentally and socially. During this time $20 \%$ of final adult height and $50 \%$ of adult weight are obtained. Anemia is a widespread nutritional problem which is more commonly seen in women and children affecting their most crucial periods like during pregnancy and growth respectively.

The prevalence of micronutrient deficiencies in rural area was as high as 25 per cent as reported by Choudhary et al with high prevalence of anemia, more among girls, ranging from 30-82 per cent $^{5}$. Anemic adolescent mothers are at a high risk of miscarriage, maternal mortality and still births; also, low birth weight babies with low iron reserves. Poor nutritional status of adolescents is an outcome of socio-cultural, economic and public policies relating to household food security compounded by behaviouraldimensions ${ }^{6}$.

\section{MATERIALS AND METHODS}

\section{Study Type}

This is the community based descriptive cross sectional study. The present study was conducted on randomly selected Adolescents girls attending Zila Parishad schools in rural area of Karad district -Satara as per the inclusion and exclusion criteria. The period of data collection was from January to july 2015. The collected data was analyzed over a two month period and the report writing was completed by end of Sep 2016.

\section{Sampling method}

The estimated prevalence of anaemia among adolescent girls as per National Family Health Survey is 56\%. ${ }^{6}$ Considering $56 \%$ prevalence of adolescent anaemia and by using the formula $\mathrm{n}=$ $4 \mathrm{pq} / \mathrm{l}^{2}$ and the allowable error of $5 \%$, the estimated sample size was 380. Participants were included in the study after taking their voluntary informed consent of their parent.

\section{Study Tools}

A predesigned semi-structured questionnaire was prepared based on the review of literature on Adolescent girl's anemia. A detailed history and physical examination was done according to a predesigned proforma to elicit various nutritional and socioeconomic factors. Age of the child was recorded in completed years. The questionnaire included the information regarding age, religion, occupation, type of family, education of participants, mother's education and father's education. It also included information regarding per capita income, socio-economic status, personal history of diet and nutritional history, past history of deworming and family history. It also included information about menstruation, height, weight, BMI, and clinical features of anemia. Under complete aseptic precautions around 3-4 cc blood was drawn from the cubital vein using a $5 \mathrm{cc}$ syringe. The blood was then inserted into two vacutainers- one with K2 EDTA having blue cap for complete blood count and another with red cap for serum investigations. Proper labeling was done to each vacutainer and then the EDTA vacutainer was send to pathology laboratory for complete blood count (CBC) and serum vacutainer was send to bio-chemistry laboratories for serum investigation of iron profile. CBC was done by using a automatic analyser of model name SYSMEX manufacture name TRANSASIA and model number of XS800i. For iron profile serum was separated by centrifuging the plain red cap vacutainer at 3000 revolutions per minute for $5 \mathrm{~min}$. MEDICON reagents are used for quantitative determination of 
unsaturated iron binding capacity and iron in serum with becman coulter. The information regarding laboratory parameters like Hemoglobin, peripheral blood smear, MCV (Mean Corpuscular Volume), MCHC (Mean Corpuscular Haemoglobin Concentration), $\mathrm{MCH}$ (Mean Corpuscular Haemoglobin), serum iron, Total Iron Binding Capacity (TIBC), serum transferring saturation were also noted.

\section{Ethical Issues}

First of all, the study protocol was approved by the Scientific and Ethical Committee of the Institution. All the participants and their parents were also informed about the study procedure and the information required from them for the study. A voluntary informed written consent was taken from the participant parent and those who consented were included in the study. A strict confidentiality was maintained about the personal details of the participants and information related to the study.

\section{Statistical Analysis}

Data management and analysis was done using Microsoft excel and Epi-info software. The frequency distribution and graph were prepared for the variables. The categorical variables were assessed using Pearson chi-square. The test was considered significant only if the $\mathrm{p}$ value comes out to be less than 0.05 .

\section{RESULTS}

Among 380 adolescent girls, hemoglobin concentration was less than $12 \mathrm{gm} / \mathrm{dl}$ among 172 (45.3\%) girls which was classified as anemia. 91 $(52.9 \%)$ girls were classified as having mild anemia (Hb- 11.0 to $11.9 \mathrm{gm} / \mathrm{dl}), 69(40.1 \%)$ had moderate anemia (Hb- 8.0 to $10.9 \mathrm{gm} / \mathrm{dl})$ and 12 (7.0\%) girls had severe anemia (Hb- less than 8 $\mathrm{gm} / \mathrm{dl})$. Religion wise distribution of study participants showed that there was no significant difference in frequencies between anemic and non-anemic girls which shows that there isn't significant association between anemia groups and religion groups. Education level and type of family were not significantly associated with anemia groups. No significant association was found between anemia groups and socioeconomic variables like father's education, mother's education and father's occupation except mother's occupation which is significant ( $p$ 0.0457).

Prevalence of anemia increases with increase in socio-economic class of study participants. Out of all anemic girls, 90 (52.3\%) girls were from socioeconomic class $\mathrm{V}$ (Lower lower class). In comparison out of all non-anemic girls, 79 (38.0\%) girls were from socioeconomic class V. The association between anemia groups and SE class was found statistically significant. ( $p$ 0.0186). Out of all anemic girls $(n=172), 98$ (57.0\%) were vegetarian by diet. In comparison, out of all non-anemic girls $(n=208), 86(41.3 \%)$ were vegetarian by diet. Thus prevalence of anemia was more in vegetarian person in comparison to non-vegetarian person. The association was found statistically significant. ( $p$ 0.002)

Prevalence of anemia was more in girls without history of deworming in comparison to with history of deworming. Out of all anemic girls $(\mathrm{n}=172)$, only $52(30.2 \%)$ had history of deworming in comparison to 124 (59.6\%) nonanemic girls. The association was found statistically significant ( $\mathrm{p}<0.001)$. Prevalence of anemia was more in girls in whom menstruation had started in comparison to those in whom menstruation was still not started. The association between anemia groups and history of menstruation was found statistically significant ( $\mathrm{p}$ 0.010). Among anemic girls, 106 (71.6\%) girls had irregular menstruation in comparison to 80 (51.0\%) among non-anemic girls. Association between anemia and menstruation was found statistically significant. $(\mathrm{p}<0.001)$. Association between anemia and inter-menstruation interval was found statistically insignificant. (p 0.2124). Prevalence of anemia was higher in participant who have heavy blood loss during menstruation. Association between anemia and amount of blood 


\section{JMSCR Vol||04||Issue||12||Page 14862-14870||December}

loss was found statistically significant. $(\mathrm{p}<0.001)$. Among anemic girls, 58 (39.2\%) had more than 5 days of blood flow during menses in comparison to $15(9.6 \%)$ girls in non-anemic group. Associations between anemia and days of blood flow \& associated clots were found statistically significant. $\quad(\mathrm{p}<0.001)$ Among anemic participants, $84(48.8 \%)$ were underweight and 88 (51.2\%) were normal weight. Among anemic participants, $63(30.3 \%)$ were underweight and $145(69.7 \%)$ were normal weight. Associations between anemia groups and BMI groups was found statistically significant. $(\mathrm{p}<0.001$. Mean differences of $\mathrm{MCH}$ (mean corpuscular haemoglobin), MCHC(mean corpuscular haemoglobin concentration) and $\mathrm{MCV}$ (mean corpuscular volume) were statistically significant among anemic and non-anemic. $(\mathrm{p}<0.001)$. Mean differences of Serum iron, TIBC (Total Iron-
Binding Capacity), Transferrin saturation, Serum ferritin and platelet count were statistically significant among anemic and non-anemic. $(\mathrm{p}<$ $0.001)$.

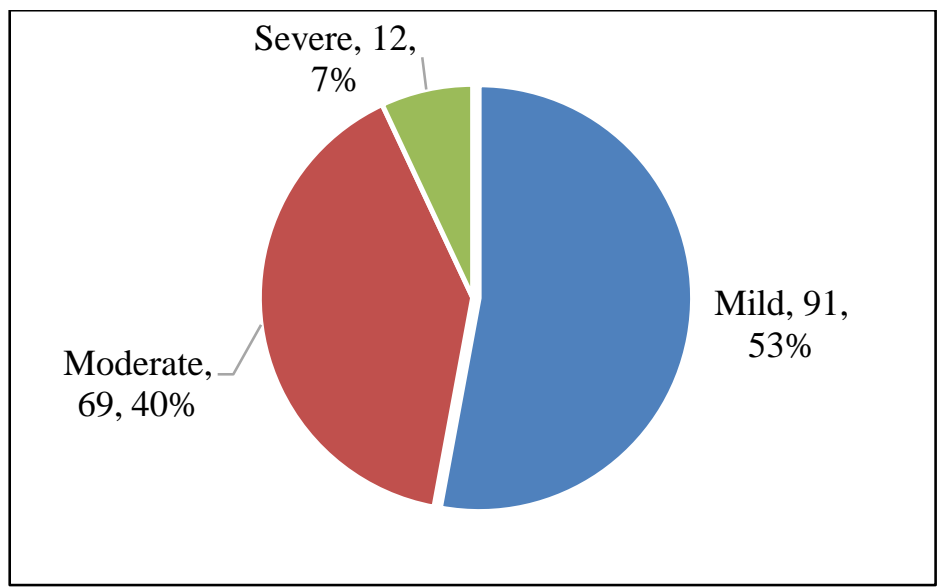

Figure: Grading of anemia according study participants

Table : Association between anemia categories and socio-demographic characteristics

\begin{tabular}{|c|c|c|c|c|c|}
\hline \multirow[b]{2}{*}{ Characteristics } & \multicolumn{2}{|c|}{ Anemic $(n=172)$} & \multicolumn{2}{|c|}{ Non Anemic $(n=208)$} & \multirow[t]{2}{*}{$\mathrm{P}$ value* } \\
\hline & Frequency & Percentage & Frequency & Percentage & \\
\hline \multicolumn{6}{|l|}{ Age } \\
\hline$<12$ years & 16 & $9.3 \%$ & 21 & $10.1 \%$ & 0.8174 \\
\hline 12-14 years & 72 & $41.9 \%$ & 91 & $43.8 \%$ & \\
\hline 14-16 years & 75 & $43.6 \%$ & 89 & $42.8 \%$ & \\
\hline$>16$ years & 9 & $5.2 \%$ & 7 & $3.4 \%$ & \\
\hline Age (Mean + SD) & 13.8 & 2.41 & 14.2 & 2.48 & \\
\hline \multicolumn{6}{|l|}{ Religion } \\
\hline Hindu & 136 & $79.1 \%$ & 165 & $79.3 \%$ & 0.9932 \\
\hline Muslim & 27 & $15.7 \%$ & 31 & $14.9 \%$ & \\
\hline Christian & 6 & $3.5 \%$ & 8 & $3.8 \%$ & \\
\hline Others & 3 & $1.7 \%$ & 4 & $1.9 \%$ & \\
\hline \multicolumn{6}{|l|}{ Education } \\
\hline Literate & $\mathbf{0}$ & $0.0 \%$ & $\mathbf{0}$ & $0.0 \%$ & 0.0800 \\
\hline Primary & 34 & $19.8 \%$ & 57 & $27.4 \%$ & \\
\hline Secondary & 138 & $80.2 \%$ & 151 & $72.6 \%$ & \\
\hline High school and Above & $\mathbf{0}$ & $0.0 \%$ & $\mathbf{0}$ & $0.0 \%$ & \\
\hline \multicolumn{6}{|l|}{ Type of family } \\
\hline Nuclear & 80 & $46.5 \%$ & 103 & $49.5 \%$ & 0.5592 \\
\hline Joint & 92 & $53.5 \%$ & 105 & $50.5 \%$ & \\
\hline
\end{tabular}

\footnotetext{
* Chi square test;
} 
Table : Association between anemia categories and socio-economic characteristics

\begin{tabular}{|l|c|c|c|c|c|}
\hline \multirow{2}{*}{ Characteristics } & \multicolumn{2}{|c|}{ Anemic $(\mathrm{n}=172)$} & \multicolumn{2}{c}{ Non Anemic $(\mathrm{n}=208)$} & \multirow{2}{*}{ P value* } \\
\cline { 2 - 5 } & Frequency & Percentage & Frequency & Percentage & \\
\hline Mother's Education & & & & & \\
\hline Literate & $\mathbf{4 9}$ & $\mathbf{2 8 . 5 \%}$ & $\mathbf{7 7}$ & $\mathbf{3 7 . 0 \%}$ & $\mathbf{0 . 1 5 4 3}$ \\
\hline Primary & $\mathbf{5 2}$ & $\mathbf{3 0 . 2 \%}$ & $\mathbf{6 0}$ & $\mathbf{2 8 . 8 \%}$ & \\
\hline Secondary & $\mathbf{2 3}$ & $\mathbf{1 3 . 4 \%}$ & $\mathbf{3 2}$ & $\mathbf{1 5 . 4 \%}$ & \\
\hline High school & $\mathbf{3 2}$ & $\mathbf{1 8 . 6 \%}$ & $\mathbf{2 9}$ & $\mathbf{1 3 . 9 \%}$ & \\
\hline PUC/graduate & $\mathbf{1 6}$ & $\mathbf{9 . 3 \%}$ & $\mathbf{9}$ & $\mathbf{4 . 3 \%}$ & \\
\hline Post graduate & $\mathbf{0}$ & $\mathbf{0 . 0 \%}$ & $\mathbf{1}$ & $\mathbf{0 . 5 \%}$ & \\
\hline Father's Education & & & & & \\
\hline Lliterate & $\mathbf{3 7}$ & $\mathbf{2 1 . 5 \%}$ & $\mathbf{5 0}$ & $\mathbf{2 4 . 0 \%}$ & $\mathbf{0 . 3 6 5 7}$ \\
\hline Primary & $\mathbf{4 0}$ & $\mathbf{2 3 . 3 \%}$ & $\mathbf{3 2}$ & $\mathbf{1 5 . 4 \%}$ & \\
\hline Secondary & $\mathbf{5 1}$ & $\mathbf{2 9 . 7 \%}$ & $\mathbf{7 8}$ & $\mathbf{3 7 . 5 \%}$ & \\
\hline High school & $\mathbf{3 0}$ & $\mathbf{1 7 . 4 \%}$ & $\mathbf{3 1}$ & $\mathbf{1 4 . 9 \%}$ & \\
\hline PUC/graduate & $\mathbf{1 0}$ & $\mathbf{5 . 8 \%}$ & $\mathbf{1 2}$ & $\mathbf{5 . 8 \%}$ & \\
\hline Post graduate & $\mathbf{4}$ & $\mathbf{2 . 3 \%}$ & $\mathbf{5}$ & $\mathbf{2 . 4 \%}$ & \\
\hline Father occupation & & & & & \\
\hline Agriculture & $\mathbf{6 7}$ & $\mathbf{3 9 . 0 \%}$ & $\mathbf{8 9}$ & $\mathbf{4 2 . 8 \%}$ & $\mathbf{0 . 4 2 5 7}$ \\
\hline Labourer & $\mathbf{6 2}$ & $\mathbf{3 6 . 0 \%}$ & $\mathbf{7 8}$ & $\mathbf{3 7 . 5 \%}$ & \\
\hline Service & $\mathbf{2 3}$ & $\mathbf{1 3 . 4 \%}$ & $\mathbf{1 8}$ & $\mathbf{8 . 7 \%}$ & \\
\hline Business & $\mathbf{1 5}$ & $\mathbf{8 . 7 \%}$ & $\mathbf{1 3}$ & $\mathbf{6 . 3 \%}$ & \\
\hline Others & $\mathbf{5}$ & $\mathbf{2 . 9 \%}$ & $\mathbf{1 0}$ & $\mathbf{4 . 8 \%}$ & \\
\hline Mother occupation & & & & & \\
\hline House wife & $\mathbf{8 8}$ & $\mathbf{5 1 . 2 \%}$ & $\mathbf{1 2 2}$ & $\mathbf{5 8 . 7 \%}$ & $\mathbf{0 . 0 4 5 7}$ \\
\hline Laborer & $\mathbf{6 5}$ & $\mathbf{3 7 . 8 \%}$ & $\mathbf{7 0}$ & $\mathbf{3 3 . 7 \%}$ & \\
\hline Service & $\mathbf{5}$ & $\mathbf{2 . 9 \%}$ & $\mathbf{8}$ & $\mathbf{3 . 8 \%}$ & \\
\hline Others & $\mathbf{1 4}$ & $\mathbf{8 . 1 \%}$ & $\mathbf{8}$ & $\mathbf{3 . 8 \%}$ & \\
\hline Chi square test & & & & & \\
\hline
\end{tabular}

* Chi square test

Table : Association between anemia categories and socioeconomic class

\begin{tabular}{|l|c|c|c|c|c|}
\hline \multirow{2}{*}{ Characteristics } & \multicolumn{2}{|c|}{ Anemic $(\mathrm{n}=172)$} & \multicolumn{2}{c|}{ Non Anemic (n=208) } & \multirow{2}{*}{ P value } \\
\cline { 2 - 5 } & Frequency & Percentage & Frequency & Percentage & \\
\hline Socioeconomic classification & & & & & \\
\hline Class II & $\mathbf{1 0}$ & $\mathbf{5 . 8 \%}$ & $\mathbf{2 4}$ & $\mathbf{1 1 . 5 \%}$ & $\mathbf{0 . 0 1 8 6}$ \\
\hline Class III & $\mathbf{2 5}$ & $\mathbf{1 4 . 5 \%}$ & $\mathbf{4 3}$ & $\mathbf{2 0 . 7 \%}$ & \\
\hline Class IV & $\mathbf{4 7}$ & $\mathbf{2 7 . 3 \%}$ & $\mathbf{6 2}$ & $\mathbf{2 9 . 8 \%}$ & \\
\hline Class V & $\mathbf{9 0}$ & $\mathbf{5 2 . 3 \%}$ & $\mathbf{7 9}$ & $\mathbf{3 8 . 0 \%}$ & \\
\hline
\end{tabular}

*Chi square test

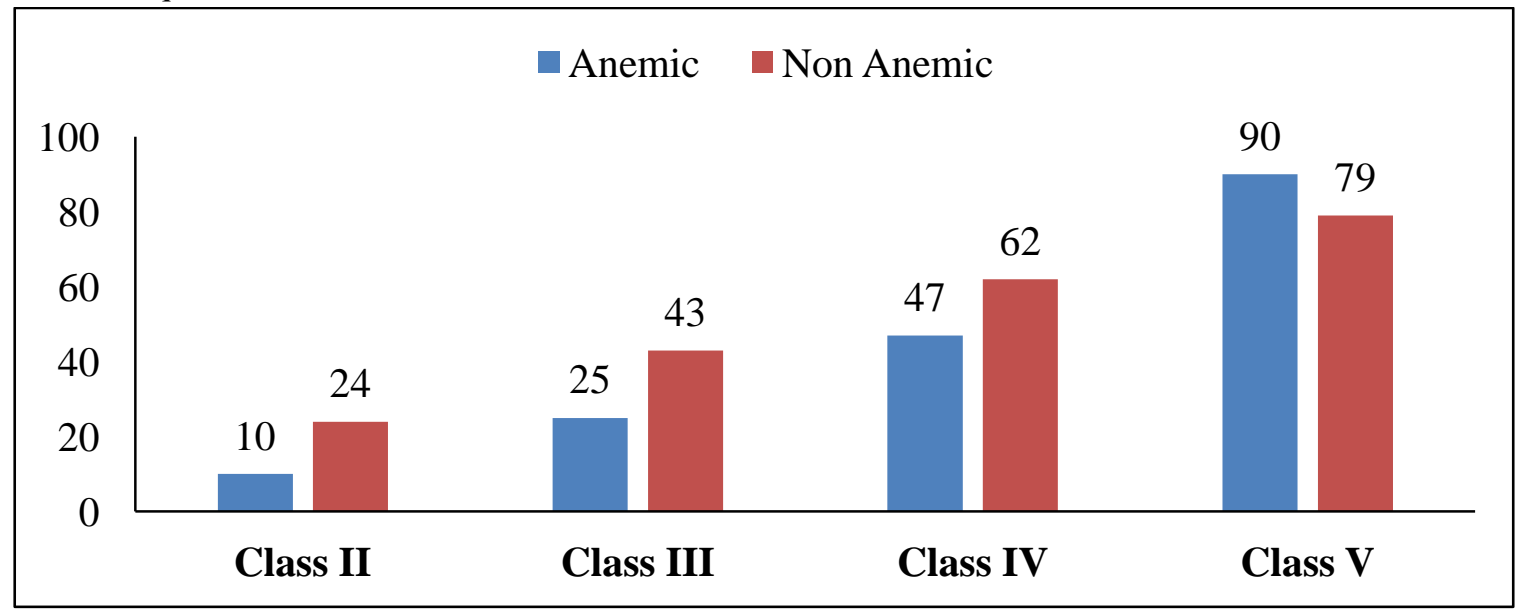

Figure: Association between anemia categories and socioeconomic class 
Table : Association between anemia categories and diet, deworming \& menstruation

\begin{tabular}{|l|l|l|l|l|l|}
\hline \multirow{2}{*}{ Characteristics } & \multicolumn{2}{|l|}{ Anemic $(\mathrm{n}=172)$} & \multicolumn{2}{l|}{ Non Anemic $(\mathrm{n}=208)$} & \multirow{2}{*}{ P value* } \\
\cline { 2 - 5 } & Frequency & Percentage & Frequency & Percentage & \\
\hline Diet & & & & & \\
\hline Vegetarian & $\mathbf{9 8}$ & $\mathbf{5 7 . 0 \%}$ & $\mathbf{8 6}$ & $\mathbf{4 1 . 3 \%}$ & $\mathbf{0 . 0 0 2}$ \\
\hline Non vegetarian & $\mathbf{7 4}$ & $\mathbf{4 3 . 0 \%}$ & $\mathbf{1 2 2}$ & $\mathbf{5 8 . 7 \%}$ & \\
\hline H/O deworming & & & & & \\
\hline Yes & $\mathbf{5 2}$ & $\mathbf{3 0 . 2 \%}$ & $\mathbf{1 2 4}$ & $\mathbf{5 9 . 6 \%}$ & $<\mathbf{0 . 0 0 1}$ \\
\hline No & $\mathbf{1 2 0}$ & $\mathbf{6 9 . 8 \%}$ & $\mathbf{8 4}$ & $\mathbf{4 0 . 4 \%}$ & \\
\hline Menstruation started & & & & & \\
\hline Yes & $\mathbf{1 4 8}$ & $\mathbf{8 6 . 0 \%}$ & $\mathbf{1 5 7}$ & $\mathbf{7 5 . 5 \%}$ & $\mathbf{0 . 0 1 0}$ \\
\hline No & $\mathbf{2 4}$ & $\mathbf{1 4 . 0 \%}$ & $\mathbf{5 1}$ & $\mathbf{2 4 . 5 \%}$ & \\
\hline * Chi square test & & & & &
\end{tabular}

Table : Association between anemia categories and menstruation characteristics

\begin{tabular}{|l|c|c|c|c|c|}
\hline \multirow{2}{*}{ Characteristics } & \multicolumn{2}{|c|}{ Anemic $(\mathrm{n}=172)$} & \multicolumn{2}{c|}{ Non Anemic $(\mathrm{n}=208)$} & \multirow{2}{*}{ P value* } \\
\cline { 2 - 5 } & Frequency & Percentage & Frequency & Percentage & \\
\hline Menstruation & & & & & \\
\hline Regular & $\mathbf{4 2}$ & $\mathbf{2 8 . 4 \%}$ & $\mathbf{7 7}$ & $\mathbf{4 9 . 0 \%}$ & $<0.001$ \\
\hline Irregular & $\mathbf{1 0 6}$ & $\mathbf{7 1 . 6 \%}$ & $\mathbf{8 0}$ & $\mathbf{5 1 . 0 \%}$ & \\
\hline Inter menstrual interval & & & & & \\
\hline$<\mathbf{2 1}$ days & $\mathbf{3 8}$ & $\mathbf{2 5 . 7 \%}$ & $\mathbf{4 6}$ & $\mathbf{2 9 . 3 \%}$ & $\mathbf{0 . 2 1 2 4}$ \\
\hline $\mathbf{2 1}-\mathbf{3 5}$ days & $\mathbf{6 7}$ & $\mathbf{4 5 . 3 \%}$ & $\mathbf{7 9}$ & $\mathbf{5 0 . 3 \%}$ & \\
\hline$>\mathbf{3 5}$ days & $\mathbf{4 3}$ & $\mathbf{2 9 . 1 \%}$ & $\mathbf{3 2}$ & $\mathbf{2 0 . 4 \%}$ & \\
\hline Amount of blood flow & & & & & \\
\hline Scanty & $\mathbf{2 1}$ & $\mathbf{1 4 . 2 \%}$ & $\mathbf{3 2}$ & $\mathbf{2 0 . 4 \%}$ & $<\mathbf{0 . 0 0 1}$ \\
\hline Moderate & $\mathbf{7 4}$ & $\mathbf{5 0 . 0 \%}$ & $\mathbf{1 0 6}$ & $\mathbf{6 7 . 5 \%}$ & \\
\hline Heavy & $\mathbf{5 3}$ & $\mathbf{3 5 . 8 \%}$ & $\mathbf{1 9}$ & $\mathbf{1 2 . 1 \%}$ & \\
\hline Days of blood flow $:$ & & & & & \\
\hline$<3$ days & $\mathbf{3 4}$ & $\mathbf{2 3 . 0 \%}$ & $\mathbf{5 3}$ & $\mathbf{3 3 . 8 \%}$ & $<\mathbf{0 . 0 0 1}$ \\
\hline $\mathbf{3}-\mathbf{5}$ days & $\mathbf{5 6}$ & $\mathbf{3 7 . 8 \%}$ & $\mathbf{8 9}$ & $\mathbf{5 6 . 7 \%}$ & \\
\hline$>\mathbf{5}$ days & $\mathbf{5 8}$ & $\mathbf{3 9 . 2 \%}$ & $\mathbf{1 5}$ & $\mathbf{9 . 6 \%}$ & \\
\hline Associated with clots & & & & & \\
\hline Yes & $\mathbf{8 9}$ & $\mathbf{6 0 . 1 \%}$ & $\mathbf{6 3}$ & $\mathbf{4 0 . 1 \%}$ & $<\mathbf{0 . 0 0 1}$ \\
\hline No & $\mathbf{5 9}$ & $\mathbf{3 9 . 9 \%}$ & $\mathbf{9 4}$ & $\mathbf{5 9 . 9 \%}$ & \\
\hline
\end{tabular}

* Chi square test

Table : Association between anemia categories and BMI categories and presence of pallor

\begin{tabular}{|l|c|c|c|c|c|}
\hline \multirow{2}{*}{ Characteristics } & \multicolumn{2}{|c|}{ Anemic $(\mathrm{n}=172)$} & \multicolumn{2}{c|}{ Non Anemic (n=208) } & \multirow{2}{*}{ P value } \\
\cline { 2 - 5 } & Frequency & Percentage & Frequency & Percentage & \\
\hline BMI kg/m2 & & & & & \\
\hline Under weight(<18.5) & $\mathbf{8 4}$ & $\mathbf{4 8 . 8 \%}$ & $\mathbf{6 3}$ & $\mathbf{3 0 . 3 \%}$ & $<\mathbf{0 . 0 0 1}$ \\
\hline Normal range (18.5-24.99) & $\mathbf{8 8}$ & $\mathbf{5 1 . 2 \%}$ & $\mathbf{1 4 5}$ & $\mathbf{6 9 . 7 \%}$ & \\
\hline Overweight(>25) & $\mathbf{0}$ & $\mathbf{0 . 0 \%}$ & $\mathbf{0}$ & $\mathbf{0 . 0 \%}$ & \\
\hline Pallor & & & & & \\
\hline Present & $\mathbf{1 4 6}$ & $\mathbf{8 4 . 9 \%}$ & $\mathbf{4 8}$ & $\mathbf{2 3 . 1 \%}$ & $<\mathbf{0 . 0 0 1}$ \\
\hline Absent & $\mathbf{2 6}$ & $\mathbf{1 5 . 1 \%}$ & $\mathbf{1 6 0}$ & $\mathbf{7 6 . 9 \%}$ & \\
\hline
\end{tabular}

* Chi square test 


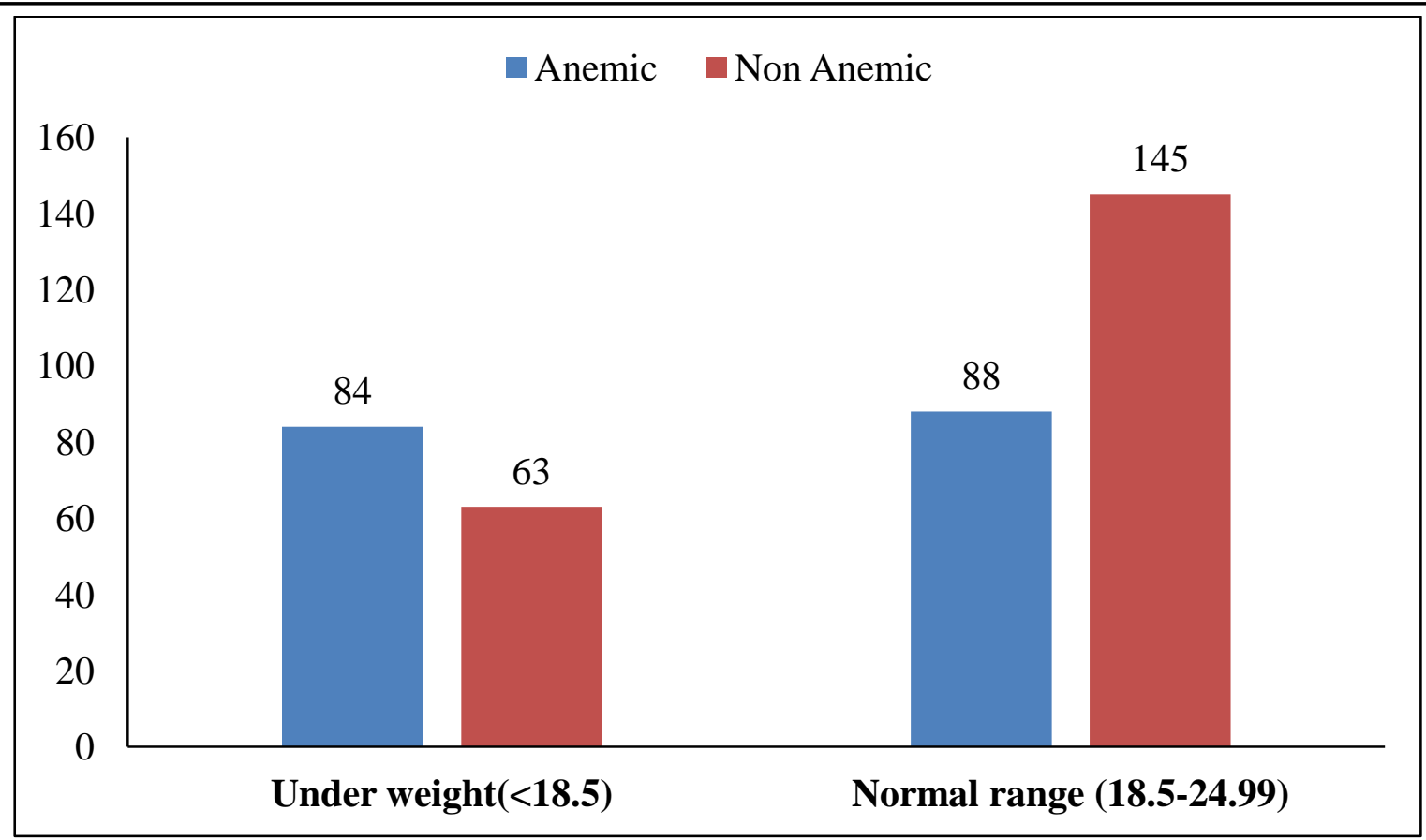

Figure: Association between anemia categories and BMI categories

Table : Association between anemia categories and laboratory indicators

\begin{tabular}{|l|c|c|c|c|c|}
\hline \multirow{2}{*}{ Characteristics } & \multicolumn{2}{|c|}{ Anemic $(\mathrm{n}=172)$} & \multicolumn{2}{|c|}{ Non Anemic $(\mathrm{n}=208)$} & \multirow{2}{*}{ P value* } \\
\cline { 2 - 5 } & Mean & SD & Mean & SD & \\
\hline MCH (mean corpuscular haemoglobin) picograms/cell & $\mathbf{2 1 . 1}$ & $\mathbf{3 . 6 8}$ & $\mathbf{3 0 . 2}$ & $\mathbf{5 . 2 7}$ & $<\mathbf{0 . 0 0 1}$ \\
\hline MCHC(mean corpuscular haemoglobin concentration) g/dl & $\mathbf{2 5 . 4}$ & $\mathbf{4 . 4 3}$ & $\mathbf{3 4 . 5}$ & $\mathbf{6 . 0 2}$ & $<\mathbf{0 . 0 0 1}$ \\
\hline MCV(mean corpuscular volume) fL & $\mathbf{6 5 . 7}$ & $\mathbf{1 1 . 4 6}$ & $\mathbf{8 8 . 9}$ & $\mathbf{1 5 . 5 1}$ & $<\mathbf{0 . 0 0 1}$ \\
\hline Serum iron $\mu$ g/dL & $\mathbf{2 7 . 3}$ & $\mathbf{4 . 7 6}$ & $\mathbf{9 4 . 4}$ & $\mathbf{1 6 . 4 7}$ & $<\mathbf{0 . 0 0 1}$ \\
\hline TIBC - Total Iron-Binding Capacity mcg/dl & $\mathbf{5 0 3 . 2}$ & $\mathbf{8 7 . 7 7}$ & $\mathbf{3 6 7 . 7}$ & $\mathbf{6 4 . 1 3}$ & $<\mathbf{0 . 0 0 1}$ \\
\hline Transferrin saturation & $\mathbf{4 . 1}$ & $\mathbf{0 . 7 2}$ & $\mathbf{2 . 9}$ & $\mathbf{0 . 5 1}$ & $<\mathbf{0 . 0 0 1}$ \\
\hline Serum ferritin microgram/L & $\mathbf{3 2 . 1}$ & $\mathbf{5 . 6 0}$ & $\mathbf{1 5 8 . 1}$ & $\mathbf{2 7 . 5 8}$ & $<\mathbf{0 . 0 0 1}$ \\
\hline platelet count & $\mathbf{5 0 5 3 6 0}$ & $\mathbf{8 8 1}$ & $\mathbf{3 5 0 6 7 9}$ & $\mathbf{6 1 1}$ & $<\mathbf{0 . 0 0 1}$ \\
\hline
\end{tabular}

*Unpaired t test

\section{DISCUSSION}

In the study conducted by Siddharam SM et al ${ }^{7}$, Out of 314 subjects, 142 (45.2\%) were found to be anemic, of which $57(40.14 \%)$ had mild anemia (Hb 10.9-11gm \%), 78 (54.92\%) had moderate anemia ( $\mathrm{Hb} 10.9-8 \mathrm{gm} \% \%)$, and 7 (4.92\%) had severe anemia $(\mathrm{Hb}<8 \mathrm{gm} \%)$ which is similar to our findings. ${ }^{8} \mathrm{WHO} / \mathrm{UNICEF}$ has suggested that the problem of anaemia is of very high magnitude in a community when prevalence rate exceeds 40\%. ${ }^{9}$ Sanjeev et al reported that the prevalence of anaemia was high among late adolescents as compared to early and mid adolescents. Hashizume et al also found that the high iron intake was significantly associated with decrease prevalence of anaemia. ${ }^{10}$ In the study conducted by Siddharam SM et al ${ }^{7}$, in anemic group $85 \%$ participants had iron deficiency and $15 \%$ does not have iron deficiency while in non-anemic group 49.5\% participants had iron deficiency and 50.5\% does not have iron deficiency.

In the study conducted by Siddharam SM et al ${ }^{7}$, in anemic group $8.4 \%$ participants belong socioeconomic class II, $26 \%$ participants belong socioeconomic class III, $33 \%$ participants belong socioeconomic class IV and $32.4 \%$ participants belong socio-economic class $\mathrm{V}$ while in anemic group 9.3\% participants belong socio-economic class II, $18 \%$ participants belong socio-economic class III, $36 \%$ participants belong socio-economic class IV 
and $36.6 \%$ participants belong socio-economic class V. In the study conducted by Pattnaik $\mathrm{S}$ et al 11, as per modified Prasad's classification and taking consumer price index of April 2012 into consideration, $74.2 \%$ of the families belonged to lower socio-economic group and $25.8 \%$ of the families belonged to middle socio-economic group. In the study conducted by R. Gawarika et al ${ }^{12}$, The overall percent prevalence of anaemia among the adolescent girls of weaker economic group was $96.5 \%$ and among girls of middle or higher middle income group was $65.18 \%$. Kaur S et al observed similar findings that girls with lower socio-economic status were associated with increased likelihood of anaemia compared to girls with higher socioeconomic status. ${ }^{13}$ In the study conducted by Premalatha T et al, ${ }^{14} 31.5 \%$ of those belonged to families earning between INR 30005000 .

In the study conducted by Pattnaik $\mathrm{S}$ et al ${ }^{11}$, among those who had $\mathrm{H} / \mathrm{O}$ worm Infestation 83.3\% participants were anemic while among those who does not had H/O worm Infestation $76.6 \%$ participants were anemic. There was no significant association found between $\mathrm{H} / \mathrm{O}$ worm Infestation and anemia.

In the study conducted by Premalatha $\mathrm{T}$ et al, ${ }^{14}$ $86.75 \%$ of participants had attained menarche, $13.3 \%$ had high menstrual bleeding. In the present study, among anemic girls, 58 (39.2\%) had more than 5 days of blood flow during menses in comparison to $15(9.6 \%)$ girls in non-anemic group. Associations between anemia and days of blood flow \& associated clots were found statistically significant $(\mathrm{p}<0.001)$.In the study conducted by Pattnaik $S$ et al ${ }^{11}$, among those who had heavy menstrual flow $89.4 \%$ participants were anemic while among those who had normal menstrual flow $63.6 \%$ participants were anemic. There was significant association found between menstrual flow and anemia. In the present study, prevalence of anemia was higher in participant who has heavy blood loss during menstruation. Association between anemia and amount of blood loss was found statistically significant $(\mathrm{p}<0.001)$.
Similar findings was also found by Kaur S et al ${ }^{13}$. In our study we have found a presence of anaemia was significantly higher among adolescent girls with undernutrition $(\mathrm{P}=0.024)$. Sidharam et al also reported the similar association between anaemia and BMI. ${ }^{7}$

\section{CONCLUSIONS AND RFECOMENDA- TIONS}

It is concluded from the present study that $45.3 \%$ of the adolescent girls are suffering from anemia. Among the factors low socio-economic status, vegetarian diet, history of deworming, presence of menstruation, irregular menstruation, heavy amount of bleeding during menstruation, more than 5 days of menstruation, menstruation associated with blood clots and underweight girls are significantly associated with the presence of anemia. From these results we recommend that adolescent girls should be dewormed every six months, iron and folic acid tablets should be supplemented under National Iron plus Initiative programme (NIPI), nutritional education should be given to these girls and efforts should be taken to take care of nutritional demand of these adolescent girls by various programme like midday meal programme of by food fortification with iron supplements.

\section{REFERENCE}

1. Irwin JJ, Kirchner JT. Anaemia in children American Family Physician 2001;64 (8):1379-86.

2. International Institute for Population Sciences (IIPS) and Macro International. 2007. National Family Health Survey (NFHS-3), 2005-06: India: Volume I. Mumbai: IIPS.

3. National Nutrition Monitoring Bureau, Diet and Nutritional Status of Rural Population and Prevalence of Hypertension among adults in Rural Areas, NNMB Technical Report No. 24, 2006, NNMB, NIN, Hyderabad. 
4. Kotecha PV. Nutritional Anemia in Young Children with focus on Asia and India. Indian Journal of Community Medicine. 2011; 36(1):8-16.

5. Lokeshwara M.R, Shah NK, Agarwal B, SachdevaA.Ed. Indian Academy of Pediatrics. IAP Specialty Series on Pediatric Hematology and Oncology (Under IAP President Action Plan. 2006; 36.

6. Haboubi GJ, Shaikh RB. A comparison of the nutritional status of adolescents from selected schools of South India and UAE : a crosssectional study. Indian $J$ Community Med 2009; 34 : 108-11.

7. Siddharam $\mathrm{S} M$, Venketesh $\mathrm{G} \mathrm{M}$, Thejeshwari H L. A Study of Anemia Among Adolescent Girls in Rural Area of Hassan district, Karnataka, South India. International Journal of Biological \& Medical Research. 2011; 2(4): 922 - 924.

8. Chaturvedi S, Kapil U, Gnanasekaran N, Sachdev HPS, Pandey RM, Bhanti T. Nutrient intake amongst adolescent girls belonging to poor socio economic groups of rural area of Rajasthan. Indian Pediatr 1996; 33: 197-201

9. WHO/UNICEF: Indicators for assessing iron deficiency and strategies for its prevention. Draft based on a WHO/UNICEF Consultation, World Health Organization, Geneva, 1996.

10. Hashizume M, Shimoda T, Sasaki S, Kunili O, Caypil W, Dauletbaev D, Chiba M. Anaemia in relation to low bioavailability of dietary iron among school aged children in the Aral Sea region Kazakhstan. Int J Food Sci Nutr 2004 Feb:55(1); 37-43.

11. Pattnaik S, Patnaik L, Kumar A, Sahu T. Prevalence of Anemia among adolescent girls in a rural area of Odisha and its epidemiological correlates. INDIAN JOURNAL OF MATERNAL AND CHILD HEALTH,2012 JAN MAR;15(1); p 1-11.

12. R. Gawarika, S. Gawarika, A.K. Mishra. Prevalence of Anaemia in Adolescent Girls Belonging to Different Economic Group. Indian Journal of Community Medicine Vol. 31, No. 4, OctoberDecember, 2006; p 287-88.

13. Kaur S, Deshmukh PR, Garg BS. Epidemiological Correlates of Nutritional Anemia in Adolescent Girls of Rural Wardha. Indian journal of community medicine, 2006; 31(4):255.

14. Premalatha T, Valarmathi S, Srijayanth P, Sundar JS, Kalpana S (2012) Prevalence of Anemia and its Associated Factors among Adolescent School Girls in Chennai, Tamil Nadu, INDIA. Epidemiol 2:118. 